\title{
Mechanical Analysis of Separate Layer Injection String during Operation Condition and its Application
}

\author{
Gang Mingchao ${ }^{1 \mathrm{a}}$, Yuan Dexin ${ }^{23 b}$, You Jing ${ }^{1 \mathrm{c}}, \mathrm{Xie}^{\mathrm{W}}$ Weiyi ${ }^{1 \mathrm{~d}}$, Wang ruil ${ }^{1 \mathrm{e}}$, Wang \\ Hanxiang ${ }^{2 f}$, Zhao Wenxiu ${ }^{19}$ \\ ${ }^{1}$ Petrochina Huabei Oilfield Company, Hebei Renqiu, 062550, China \\ ${ }^{2}$ College of Electromechanical Engineering in China University of Petroleum, Shandong QingDao, \\ 266580, China
}

${ }^{3}$ Petroleum Industry Training Center in China University of Petroleum, Shandong QingDao, 266580, China

${ }^{a}$ cyy_gangmc@petrochina.com.cn, b15563442146@163.com, ccyy_youjing@petrochina.com.cn, dcyy_xiewy@petrochina.com.cn, ${ }^{\mathrm{e}}$ cyy_wr@petrochina.com.cn, f, ${ }^{\mathrm{g}} \mathrm{cyy}$ _zaowx@petrochina.com.cn

Keywords: Separate layer water injection, String mechanics, Dynamic analysis, Software development

Abstract: Separate layer water injection technic is one of the efficient measures for keeping petroleum output steady and improving oil recovery rate. In the actual operating mode, injection string which be placed in the three-dimensional wellbore may produce complicated stress and strain. Analyzing mechanical property in different conditions accurately is very important for improving design level of string and success rate of separate layer injection. Considered wellbore curvature and spatial stress status, a three-dimension mechanical analysis model of layer water injection string was established which analyzed the comprehensive influence of injection tools such as packer, water distributor, centering guide, and the mechanical continuity conditions of injection tools were established. Based on the mechanical model, considering the influence of working parameter, a mechanics behavior analysis method and procedure were proposed in the whole process of the separate injection pipe strings. In addition, the mechanical analysis software was developed using

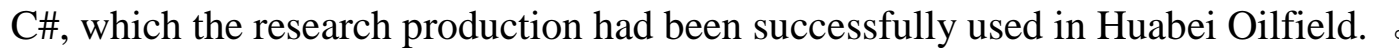

\section{Introduction}

Separate layer water injection is one of the efficient measures for keeping formation energy and improving oil recovery rate. The reasonable design of separate flooding string is the important guarantee to realize separate injection development. In the actual development, injection string which be placed in the three-dimensional wellbore may produce complicated stress and strain and be forced by a combination of a variety of load. If the string design is not reasonable, some accidents, such as the packer moving, tubing breaking off, string failing to be retrieved, leading to the failure of separate work. Combined with the actual operational mode, the accurate analysis of the mechanical properties of separate injection strings is of great significance to design injection string scientifically, optimize construction parameters reasonably and improve the working safety and success rate of separated layer water flooding works. 
Many scholars both at home and abroad researched mechanics analysis of separate injection string, having formed a variety of analysis theories and methods [1-3]. However, the dynamic analysis of separate tubing mechanical properties under various operation conditions is little being reported. On the basis of the existed research results, a three-dimensional mechanical model is established for injection strings as well as continuity conditions, tubing stress and deformation problem are discussed systematically in the process of separating, a dynamic analysis method and process are put forward, and relevant calculation software is developed and put into application.

\section{Mechanical Analysis Model of Separate Injection String}

Layering water injection string in the wellbore gets a combination of a variety of load and the stress state is complex. In order to reflect its force conditions, the injection string is put into the three-dimensional space perspective. Considering the comprehensive influence of its internal force (torque), uniform external force, pressure and friction of internal and external wellbore fluid, wellbore geometrical locus, the three-dimensional mechanical model of layering water injection string is set up.

The unit vector of tangential, normal and binormal of any point in a bit string axis are represented by $\tau, \dot{c}, \dot{b}$ respectively, the curvature, torsion and clearance between string and casing of separate injection wellbore are represented by $\begin{aligned} & \tau \\ & \tau\end{aligned} \dot{n}, \dot{b}$ respectively. The infinitesimal body on the string is taken from $s$ to $s+d s$. The deviation angle of up endpoints $\mathrm{A}$ is $\alpha_{i}$, azimuth angle is $\varphi_{i}$. The deviation angle of up endpoints B is $\alpha_{i+1}$, azimuth angle is $\varphi_{i+1}$. Assuming that string axis and wellbore geometrical locus is consistent, force analysis of infinitesimal section is shown in Fig. 1.

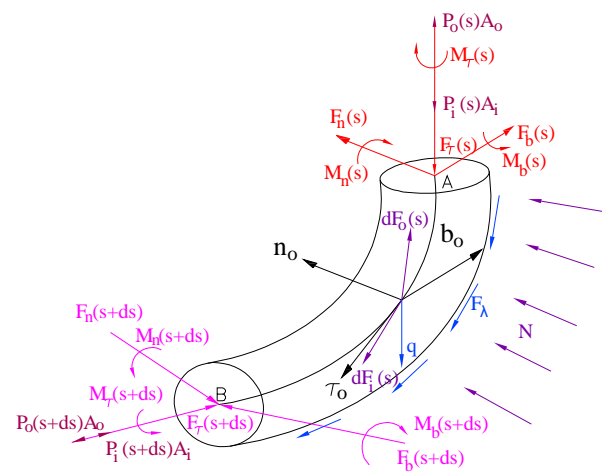

Fig.1 Mechanical analysis diagram of string infinitesimals body

The stress of separate injection string in the three-dimensional space including:

(1) The internal force and torque of injection string

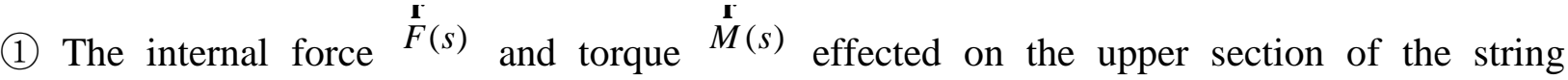
infinitesimal section

$$
\begin{aligned}
& \stackrel{\prime}{F}(s)=F_{\tau}(s) \tau_{0}^{r}+F_{n}(s) n_{0}^{r}+F_{b}(s) b_{0}^{\prime} . \\
& \dot{M}(s)=M_{\tau}(s) \tau_{0}+M_{n}(s) n_{0}+M_{b}(s) b_{0}^{\prime} .
\end{aligned}
$$


(2) The internal force ${ }^{-\mathfrak{F}(s+\mathrm{d} s)}$ and torque ${ }^{-\dot{M}(s+\mathrm{d} s)}$ effected on the lower section of the string infinitesimal section

(2) The uniform force of infinitesimal section in separate injection string

(1) The buoyant weight in unit length of separate injection string is $\stackrel{\prime}{Q}=q \dot{k}$, of which the unit is $\mathrm{N} / \mathrm{m}$;

(2) The normal positive pressure of casing wall on the string in unit length is $\dot{N}=N \cos \theta r_{0}^{r}-N \sin \theta b_{0}$, of which the unit is $\mathrm{N} / \mathrm{m}$;

(3) The viscous friction resistance produced by the interaction of internal and external fluid in unit length of string is $\left(f_{i}+f_{o}\right) \tau_{0}^{\prime}$, of which the unit is $\mathrm{N} / \mathrm{m}$;

(4) The friction force produced by wellbore wall in unit length of string is $f_{1} N \tau_{0}$, of which the unit is $\mathrm{N} / \mathrm{m}$.

Where, $q$ is the buoyant weight in unit length of string in the wellbore, $f_{1}$ is the friction coefficient between separate injection string and casing wall; , respectively, $f_{i} 、 f_{o}$ are the viscous friction resistance produced by inside and outside fluid on the injection string of unit length respectively.

(3)The pressure of internal and external fluid of string infinitesimal section [4]

Internal pressure can be equivalent to a pair of axial compressive force $\left(\stackrel{P}{P}_{i}(s)\right.$ and $-P_{i}(s+\mathrm{d} s)$ and the down distributed force $\mathrm{d} F_{i}(s)=\left(A_{i} \rho_{i} g k-f_{i}^{r} \tau_{0}\right) \mathrm{d} s$. The axial compressive force impacts on the cross section of infinitesimal section both ends and the distributed force impacts on the whole infinitesimal section.

External pressure can be equivalent to a pair of axial stretching force $\left(-{ }^{\prime} P_{o}(s)\right.$ and $\left.{ }^{\stackrel{\prime}{P}(s+\mathrm{d} s)}\right)$ and the up distributed force $\left.\stackrel{\mathrm{r}}{\mathrm{d} F_{o}(s)=\left(-A_{o} \rho_{o} g r\right.}+\frac{A_{o}}{A_{c}}\left(f_{o}+f_{c}\right) \tau_{0}\right) \mathrm{d} s$ the cross section of infinitesimal section both ends and the distributed force impacts on the whole infinitesimal section.

$\rho_{i}$ and $\rho_{o}$ are fluid density of the inside and outside string, of which the unit is $\mathrm{kg} / \mathrm{m}^{3}$. $A_{i}$ and $A_{o}$ are sectional area of inside and outside string, $A_{c}$ is annular area, of which the unit is $\mathrm{m}^{2} . f_{c}$ is the viscous friction resistance produced by annular fluid on injection string of unit length

Under the combination of various loads, the separate injection string is in balance. Thus mechanics equilibrium equation is built. The equation is: 


$$
\left\{\begin{array}{l}
\frac{\mathrm{d} F_{e \tau}(s)}{\mathrm{d} s}-k_{0} F_{n}=q_{e} \cos \alpha+f_{1} N+f_{o}+\frac{A_{o}}{A_{c}}\left(f_{o}+f_{c}\right) \\
\frac{\mathrm{d} F_{n}(s)}{\mathrm{d} s}+k_{0} F_{e \tau}-T_{0} F_{b}=N \cos \theta-q_{e} \frac{\sin \alpha}{k_{0}} \frac{\mathrm{d} \alpha}{\mathrm{d} s} \\
\frac{\mathrm{d} F_{b}(s)}{\mathrm{d} s}+T_{0} F_{n}=q_{e} \frac{\sin ^{2} \alpha}{k_{0}} \frac{\mathrm{d} \varphi}{\mathrm{d} s}-N \sin \theta \\
\frac{\mathrm{d} M_{\tau}(s)}{\mathrm{d} s}-k_{0} M_{n}=r\left(F_{n} \frac{\mathrm{d} \sin \theta}{\mathrm{d} s}+F_{b} \frac{\mathrm{d} \cos \theta}{\mathrm{d} s}\right) \\
\frac{\mathrm{d} M_{n}(s)}{\mathrm{d} s}+k_{0} M_{\tau}-T_{0} M_{b}=F_{b}-F_{e \tau} r \frac{\mathrm{d} \sin \theta}{\mathrm{d} s} \\
\frac{\mathrm{d} M_{b}(s)}{\mathrm{d} s}+T_{0} M_{n}=-F_{e \tau} r \frac{\mathrm{d} \cos \theta}{\mathrm{d} s}-F_{n}
\end{array} .\right.
$$

Where, $F_{\tau}(s), F_{n}(s), F_{b}(s), M_{\tau}(s), M_{n}(s)$ and $M_{b}(s)$ is component force and torque in the direction of $\dot{\tau}, \quad ' n, \quad \dot{b}$ of string respectively. $F_{e \tau}(s)=F_{\tau}(s)+p_{i}(s) A_{i}-p_{o}(s) A_{o}$, representing equivalent axial force.

Combined with physical equation of string:

$$
\stackrel{r}{M}(s)=-E I k_{0} b=E I r \frac{\mathrm{d}^{2} \sin \alpha}{\mathrm{d} s^{2}}{ }_{n_{0}}^{r}+E I\left(r \frac{\mathrm{d}^{2} \cos \alpha}{\mathrm{d} s^{2}}-k_{0}\right) \stackrel{r}{b_{0}}
$$

Ignoring the higher order infinitesimal, deformation differential equation of injection string in three-dimensional curved hole is got.

$$
\frac{d^{4} \theta}{d s^{4}}+\frac{d}{d s}\left[\frac{F_{e \tau}}{E I} \frac{d \theta}{d s}-2\left(\frac{d \theta}{d s}\right)^{3}\right]+\frac{f_{n}}{E I r} \sin (\theta-\psi)=0
$$

Where,

$$
\begin{aligned}
& \tan \psi=\frac{f_{1}}{f_{2}}, f_{1}=\frac{q_{e} \sin ^{2} \alpha}{k_{0}} \frac{d \varphi}{d s}, f_{2}=k_{0} F_{e \tau}+\frac{q_{e} \sin \alpha}{k_{0}} \frac{d \alpha}{d s} . \\
& f_{n}=\sqrt{f_{1}^{2}+f_{2}^{2}} .
\end{aligned}
$$

To consider a variety of practical factors such as wellbore trajectory and string load, equation (5), the three-dimensional mechanical model injection string, is established which is the foundation of analyzing points force and deformation of injection string further. 


\section{Load Analysis}

Solving equation (5), dimensionless variable $\beta=\frac{F_{e \tau}}{2 E I \mu^{2}}=\frac{F_{e \tau}}{2}\left(\frac{r}{E I f_{n}}\right)^{\frac{1}{2}}$ is introduced to simplify the equation. From the analysis, different value of $\beta$ reflects different inflexion mode of strings in the wellbore. Through calculation, when $\beta \leq 1$, string is in a stable state without buckling; when $1<\beta \leq 1.469$, string is in sinusoidal buckling state and the space configuration approximates sine curve; when $\beta>1.469$, string is in helical buckling state and the space configuration is helix.

To derivate further, ignoring the higher order infinitesimal, the following conclusions are got:

(1) The equivalent axial force of string is:

$$
\frac{\mathrm{d} F_{e \tau}(s)}{\mathrm{d} s}=q_{e} \cos \alpha-f_{1} N+f_{o}+\frac{A_{o}}{A_{c}}\left(f_{o}+f_{c}\right)
$$

Then the axial force is $F_{\tau}(s)=F_{e \tau}(s)-p_{i}(s) A_{i}+p_{o}(s) A_{o}$.

(2) After buckling, positive pressure produced by casing wall on the string is $N$. For sine buckling, $N(\mathrm{~s})=\frac{r F_{\tau}^{2}(\mathrm{~s})}{8 E I}$. For helix buckling, $N(\mathrm{~s})=\frac{r F_{\tau}^{2}(\mathrm{~s})}{4 E I}$. The bending moment of string is $M(\mathrm{~s})=\frac{r F_{\tau}(\mathrm{s})}{2}$.

\section{Deformation Analysis}

When working, because the working conditions, such as temperature, pressure, is changing, injection string will generate axial deformation. A lot of analysis and experiments show that these main factors which lead to the axial deformation of injection string are temperature effect, ballooning effect, axial force effect and buckling effect. The calculation formulas of deformation produced by various effects are shown in Table 1 . The total deformation of injection string is the algebraic sum of deformations produced by four kinds of effects.

Table1 Deformation analysis of separate layer injection string under different effects [5-7]

\begin{tabular}{ccc}
\hline Effect name & Calculation formula & Cause \\
\hline temperature effect & $u_{T}(s)=u_{T}\left(s_{0}\right)+a \int_{s_{0}}^{s}\left[T(s)-T_{1}(s)\right] d s$ & Change of temperature \\
ballooning effect & $u_{p}(s)=u_{p}\left(s_{0}\right)+\frac{2 v}{E\left(r_{o}^{2}-r_{i}^{2}\right)} \times\left[r_{o}^{2} \int_{s_{0}}^{s} P_{o}(s) d s-r_{i}^{2} \int_{s_{0}}^{s} P_{i}(s) d s\right]$ & $\begin{array}{c}\text { Change of internal and } \\
\text { external pressure }\end{array}$ \\
axial force effect & $u_{F}(s)=u_{F}\left(s_{0}\right)-\frac{1}{E\left(A_{o}-A_{i}\right)} \int_{s_{0}}^{s} F_{\tau}(s) d s$ & Axial load \\
buckling effect & $u_{s}(s)=u_{s}\left(s_{0}\right)+\int_{s_{0}}^{s}\left[r l_{o}(\cos \theta-1)+\frac{1}{2} r^{2}\left(\frac{d \theta}{d s}\right)^{2}\right] d s$ & String buckling
\end{tabular}


In the table, $\alpha$ is coefficient of thermal expansion, $1 /{ }^{\circ} \mathrm{C} ; T(s)$ is string temperature after injecting water in $s$-depth, ${ }^{\circ} \mathrm{C} ; T_{0}(s)$ is initial temperature of string when not injecting water in $s$-depth, ${ }^{\circ} \mathrm{C} ; \mathrm{v}$ is Poisson's ratio of materials.

\section{Strength Analysis}

The received stress of separate string in wellbore include radial stress, circumferential stress, axial stress and bending stress. The causes and formulas of every stress are shown in Table 2 . Under general conditions, when in the wellbore, the string is in the complicated state of three-dimensional stress, so the stress must be checked according to the Forth Strength Theory.

Table 2 Stress origin and calculation of separate layer injection string [8-10]

\begin{tabular}{ccl}
\hline Stress form & \multicolumn{1}{c}{ Calculation formula } & \multicolumn{1}{c}{ Cause } \\
\hline radial stress & $\sigma_{r}=\frac{P_{i}(\mathrm{~s}) r_{i}^{2}-P_{o}(\mathrm{~s}) r_{o}^{2}}{r_{o}^{2}-r_{i}^{2}}-\frac{r_{r}^{2} r_{o}^{2}\left[P_{i}(\mathrm{~s})-P_{o}(\mathrm{~s})\right]}{\left(r_{o}^{2}-r_{i}^{2}\right) r^{2}}$ & $\begin{array}{l}\text { Pressure of internal and external } \\
\text { string }\end{array}$ \\
circumferential stress & $\sigma_{\theta}=\frac{P_{i}(\mathrm{~s}) r_{i}^{2}-P_{o}(\mathrm{~s}) r_{o}^{2}}{r_{o}^{2}-r_{i}^{2}}+\frac{r_{i}^{2} r_{o}^{2}\left[P_{i}(\mathrm{~s})-P_{o}(\mathrm{~s})\right]}{\left(r_{o}^{2}-r_{i}^{2}\right) r^{2}}$ & $\begin{array}{l}\text { Pressure of internal and external } \\
\text { string }\end{array}$ \\
axial stress & $\sigma_{F}=-\frac{F_{\tau}(\mathrm{s})}{A_{o}-A_{i}}$ & Axial force \\
bending stress & $\sigma_{M}= \pm \frac{4 M(s) r}{\pi\left(r_{o}^{2}-r_{i}^{2}\right)}$ & Wellbore bending \\
\hline
\end{tabular}

In the table, $r_{i}, r_{o}$ is the internal and external radius; $r$ is radius in any location between $r_{i}$ and $r_{o} . M(s)$ is bending moment of string in $s$-depth, $\mathrm{N} \cdot \mathrm{m}$.

\section{Continuity Conditions of Separate Injection Tools}

Separate water injection string is composed of tubing and different kinds of separate injection tools which contain packer, water injection allocator, centralizer and slip joint. The packer is used to separate each reservoir, forming relatively independent fluid channel between string and every oil reservoir. The water injection allocator is used to control each interval water injection by its choking effect. The centralizer is used to control the radial deviation of string in the process of tripping, at the same time to protect the packer from wearing. The slip joint is used to compensate string creeping. All kinds of separate injection tools not only realize their functions but also have some effect on the mechanical properties of string. In order to form a complete mechanical model of injection string, the proper continuity conditions should be established according to the characteristics of different separate injection tools.

(1) Packer

In the packer, the displacement and internal pressure of injection string do not change suddenly, but the external pressure of up and down packer and axial force do not discontinuity. Take an infinitesimal section of packer, analyze its forces, as shown in Fig. 2. Assuming that friction force of packer on casing wall is $f, A_{c i}$ is cross-sectional area internal casing. 


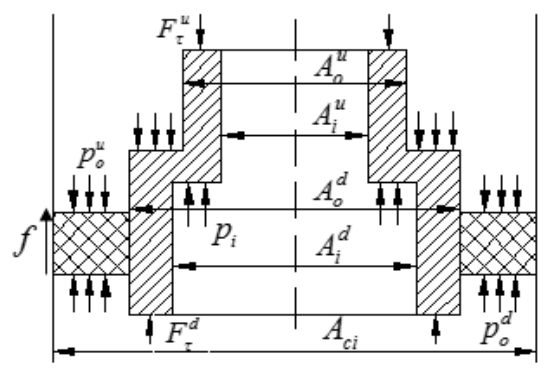

Fig.2 Mechanical analysis of packer infinitesimal section

The mechanical equilibrium equation of this location is

$$
F_{\tau}^{u}(s)+p_{i}(s)\left(A_{i}^{u}-A_{i}^{d}\right)=F_{\tau}^{d}(s)+p_{o}^{d}(s)\left(A_{c i}-A_{o}^{d}\right)-p_{o}^{u}(s)\left(A_{c i}-A_{o}^{u}\right)+f .
$$

After calculation:

$$
F_{\tau e}^{u}(s)=F_{\tau e}^{d}(s)+\left[p_{o}^{d}(s)-p_{o}^{u}(s)\right] A_{c i}+f
$$

When packer not setting, the external pressure on both sides is the same, without friction between casing and packer rubber, $F_{\tau e}^{u}(s)=F_{\tau e}^{d}(s)$, namely the equivalent axial force is continuous. After setting, $p_{o}^{d}(s) \neq p_{o}^{u}(s)$, the casing has the friction effect on packer rubber, equivalent axial force change suddenly, but at this moment packer position is fixed, $\Delta u(\mathrm{~s})=0$. Through the deformation coordination conditions, friction of casing on the packer rubber can be iterative calculated.

(2) Water injection allocator

In the water injection allocator, the displacement and internal pressure of injection string do not change suddenly, but because of choking effect, the real axial force in water distributor is in discontinuity, external pressure and internal pressure has a certain relationship. According to the throttling coefficient of distribution core the pressure value after passing water nozzle can be determined. Take an infinitesimal section of injection allocator, analyze its forces, as shown in Fig. 3 .

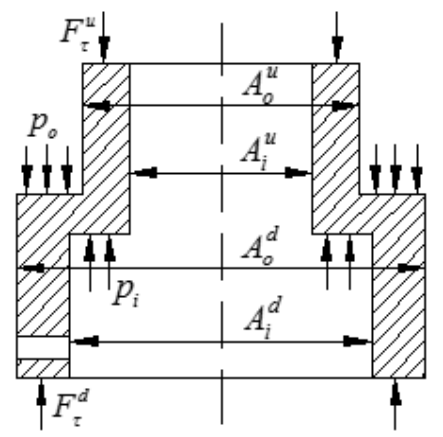

Fig.3 Mechanical analysis of water distributor infinitesimal section

The mechanical equilibrium equation of this location is 


$$
F_{\tau}^{u}(s)+p_{o}(s)\left(A_{o}^{d}-A_{o}^{u}\right)=F_{i}^{d}(s)+p_{i}(s)\left(A_{i}^{d}-A_{i}^{u}\right) .
$$

After calculation:

$$
F_{\tau e}^{u}(s)=F_{\tau e}^{d}(s)
$$

Thus, in the water distributor, equivalent axial force of injection string is continuous.

(3) Centralizer

For well righting effect, the outer diameter of centralizer is general larger. In the centralizer, the internal and external pressure of injection string does not change suddenly, so as to the displacement. That is $u_{\tau}^{u}(s)=u_{\tau}^{d}(s), p_{i}^{u}(s)=p_{i}^{d}(s), p_{o}^{u}(s)=p_{o}^{d}(s)$.

Take an infinitesimal section of centralizer, to analyze its forces, as shown in Fig. 4.

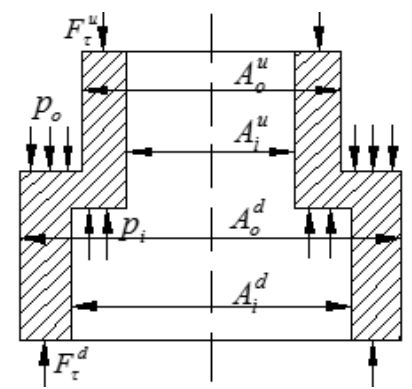

Fig.4 Mechanical analysis of centering guide infinitesimal section

The mechanical equilibrium equation of this location is

$$
F_{\tau}^{u}(s)+p_{i}(s)\left(A_{i}^{u}-A_{i}^{d}\right)=F_{\tau}^{d}(s)+p_{o}(s)\left(A_{o}^{u}-A_{o}^{d}\right) .
$$

Where, $A_{i}^{u}, A_{o}^{u}$ is inside and outside sectional area of upside string in centralizer, $A_{i}^{d}, A_{o}^{d}$ is inside and outside sectional area of centralizer.

After calculation:

$$
F_{\tau}^{u}(s)+p_{i}(s) A_{i}^{u}-p_{o}(s) A_{o}^{u}=F_{\tau}^{d}(s)+p_{i}(s) A_{i}^{d}-p_{o}(s) A_{o}^{d} .
$$

That is $F_{\tau e}^{u}(s)=F_{\tau e}^{d}(s)$.

Thus, in centralizer, the real axial force of injection string is discontinuous but the equivalent axial force is continuous.

(4) Slip joint

In separate injection string, to compensate the axial deformation of tubing, slip joint is used. In slip join, the internal and external pressure of injection string do not change suddenly but the displacement is discontinuous and so as to axial force. 
Take an infinitesimal section of slip join, to analyze its forces, as shown in Fig. 5.

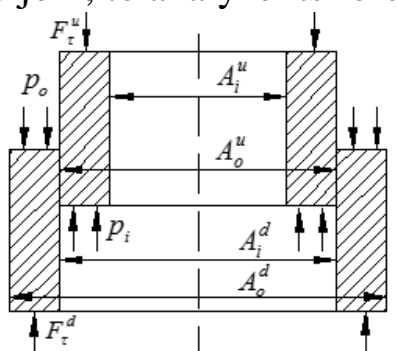

Fig.5 Mechanical analysis of expansion element infinitesimal section

Supposed that the cross-sectional area of the excircle in upper draw tube is $A_{o}^{u}$, the cross-sectional area of the excircle in lower draw tube is $A_{i}^{d}$, the mechanical equilibrium equation of this location is set up:

$$
F_{\tau}^{u}(s)+p_{o}(s)\left(A_{o}^{d}-A_{i}^{d}\right)=F_{i}^{d}(s)+p_{i}(s)\left(A_{o}^{u}-A_{i}^{u}\right) .
$$

Actual, $A_{o}^{u}=A_{i}^{d}$, After calculation:

$$
F_{\tau}^{u}(s)+p_{i}(s) A_{i}^{u}-p_{o}(s) A_{o}^{u}=F_{\tau}^{d}(s)+p_{i}(s) A_{i}^{d}-p_{o}(s) A_{o}^{d} .
$$

That is $F_{\tau e}^{u}(s)=F_{\tau e}^{d}(s)$.

Thus, in slip join, the equivalent axial force of injection string is continuous. The deformation of slip join can be calculated by the difference of deformation of tubing string up and down.

\section{A Mechanics Behavior Analysis Method Considering the Influence of Working Parameter in the Whole Process of Working}

After string being tripped and the completion of the setting, normal separate operations is turned to. There are 3 working conditions including injecting water, stop injecting, well flushing. In different conditions, the boundary condition of stress is different in injection string. During injecting water, the liquid is injected from the tubing injection, through water distributor into the wellbore and formation, and the tubing internal pressure is greater than that of outside. When stopping injecting, there is no wellhead pressure, the inside and outside of string is not connected, the inside and outside pressure of tube are not related. When well flushing, the liquid is injected from the annulus, through the flushing channel of packer to the internal tubing and then reversed to the wellhead, the tubing pressure is greater than that of internal.

The transforming of different operating mode makes the temperature and pressure change of injection strings, which produces a great impact on the mechanical properties of injection strings. Based on the mechanical model of injection strings, a mechanics analysis method of a whole process is put forward, as shown in Fig. 6. Through the two sets of circulation, the internal and external, mechanical properties of injection strings under different working conditions are iteratively computed. Inner circulation calculates the stress and deformation of pipe string in 
different depths after determining the various operation parameters. After the whole string calculation is finished, the results of the analysis are saved, as the initial values of next computation. External circulation achieves continuous analysis of different operating modes by updating operation parameters of the separate injection wells, such as wellbore temperature and pressure.

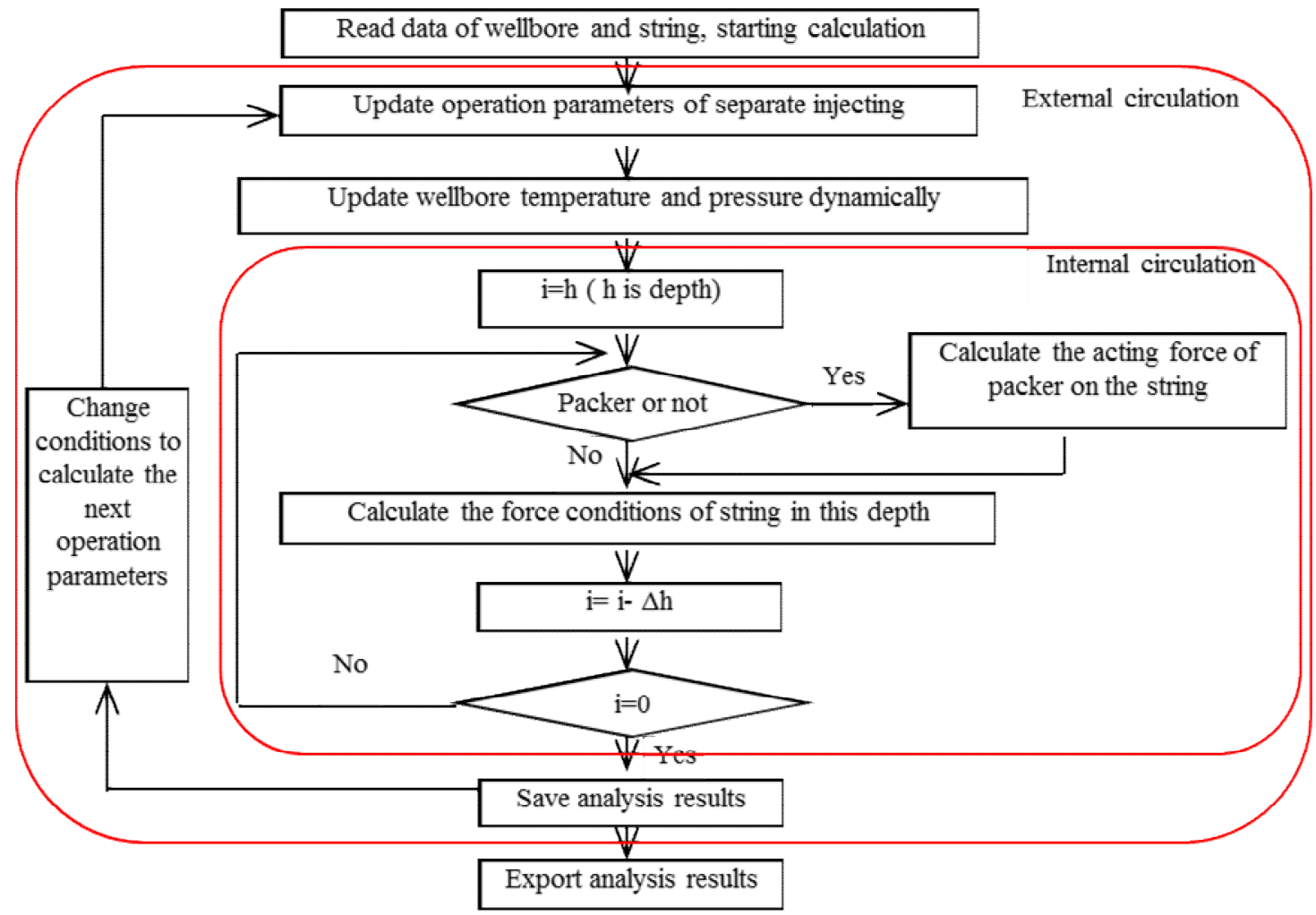

Fig.6 Dynamic analysis flow chart

\section{Software Development and Application}

According to three-dimensional mechanics analysis model of separate injection string and the mechanics behavior analysis method of different working parameters in the whole process of working, analysis software is developed by using the C\# Language. The software includes the functions data input, three-dimensional simulation of wellbore and separate injection string, mechanics analysis of separate injection string, result output, data management and so on, which can realize continuous analysis of mechanics performance in the whole operation process and evaluate safety performance of separate injection string.

The well Jin $105-12 \mathrm{X}$ is a separate injection well, of which well depth is $2,970 \mathrm{~m}$, the max deviation angle is $50.7^{\circ}$. The wellbore trajectory drew by this software is as shown in Fig. 7. The depth of the oil layer 1 is 2,543 2,586 meters, the depth of the oil layer 2 is 2,606 2,618.5 meters, the original formation pressure of this area is $25.95 \mathrm{MPa}$, wellhead average temperature for $19^{\circ} \mathrm{C}$. The one casing program is: depth of $0 \sim 113 \mathrm{~m}$, outer diameter of $339.73 \mathrm{~mm}$, wall thickness of $9.65 \mathrm{~mm}$, steel grade of J55. The other is depth of $131 \sim 2900 \mathrm{~m}$, outer diameter of $139.7 \mathrm{~mm}$, wall thickness of $7.72 \mathrm{~mm}$, steel grade of $\mathrm{N} 80$. The structure of separate injection string is: bottom screen blinding + water distributor + packer + centralizer + water distributor + packer + centralizer + safety joint + tubing anchor. 


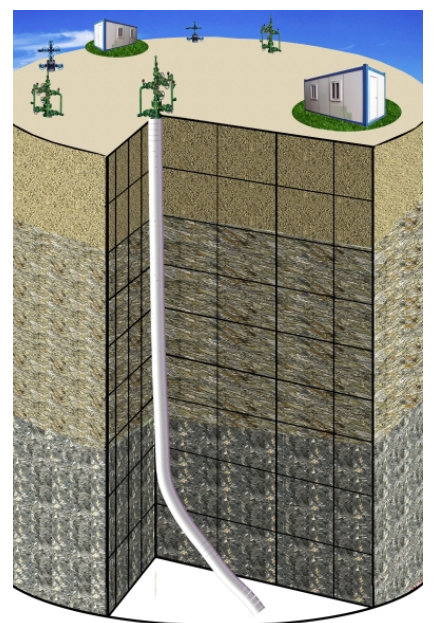

Fig.7 Well track of Jin105-12X

The working condition table is based on injection water daily table of oil field, as shown in Table 3. After finish tripping and setting, in accordance with operation parameters in Table 3, the string dynamic analysis of mechanical performance is analyzed dynamically.

Table 3 The table of layered water injection working parameter

\begin{tabular}{cccccccc}
\hline Number & Condition & Time & $\begin{array}{c}\text { Time } \\
\text { unit }\end{array}$ & $\begin{array}{c}\text { Injection } \\
\text { capacity } \\
{\left[\mathrm{m}^{3} / \mathrm{d}\right]}\end{array}$ & $\begin{array}{c}\text { Oil } \\
\text { pressure } \\
{[\mathrm{MPa}]}\end{array}$ & $\begin{array}{c}\text { Casing } \\
\text { pressure } \\
{[\mathrm{MPa}]}\end{array}$ & $\begin{array}{c}\text { Injection } \\
\text { temperature } \\
{\left[{ }^{\circ} \mathrm{C}\right]}\end{array}$ \\
\hline 1 & Injecting & 15 & day & 80 & 18.2 & 0 & 25 \\
2 & Stopping & 3 & hour & & & & \\
3 & Injecting & 21 & hour & 80 & 18.2 & 0 & 25 \\
4 & Stopping & 3 & hour & & & & \\
5 & Injecting & 10 & day & 80 & 18.5 & 0 & 25 \\
6 & Stopping & 2 & day & & & & 25 \\
7 & Flushing & 3 & hour & 380 & 5.5 & 6 & 25 \\
8 & Injecting & 10 & day & 80 & 19 & 0 & 25 \\
9 & Stopping & 7 & hour & & & & \\
10 & Injecting & 12 & hour & 80 & 20.8 & 0 & 25 \\
\hline
\end{tabular}

Figure 8 is mechanics state of injection string when the operation of this stage is over. Figure 8 is the analysis results of mechanical properties such as the maximum axial force, the maximum stress under every operating mode. From the figure 8, the maximum stress position is in the 2,017 meter apart from the wellhead. It is in building up section, so the bending moment is larger; The maximum axial force is at wellhead, and both changes suddenly at two-level of packer .The overall safety coefficient of this set of separate injection string is low. 


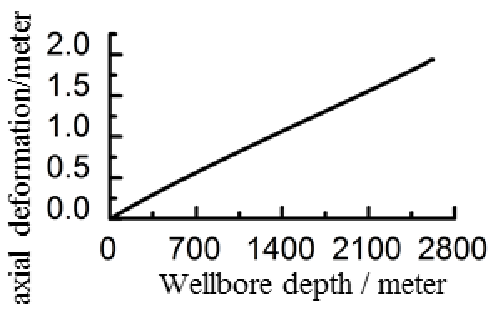

(a) Axial deformation of string

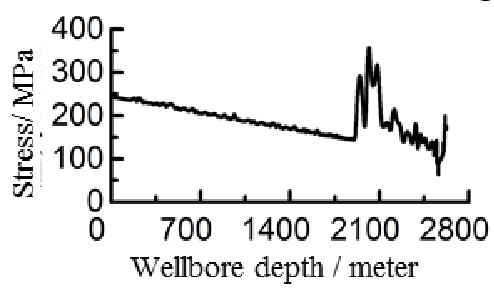

(b) Stress of string

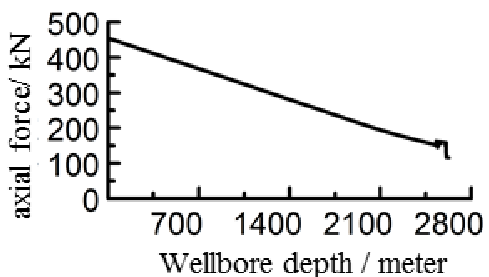

(c) Axial force of string

Fig8 The mechanical condition of layered water injection string

\section{Conclusions}

(1) Considering wellbore geometry trajectory and space force of separate injection string, a three-dimensional mechanics analysis model of separate injection string of is set up.

(2) Through analyzing the influence of separate injection tools on mechanics performance of injection string, the mechanical continuity conditions at some location such as packer, water distributor, centralizer and slip joint, is established.

(3) Put forward a mechanics behavior analysis method and procedure in the whole process of the separate injection pipe strings. Through the internal and external circulation, the continuous analysis of mechanics performance in injection string is realized.

(4) By using the C \# Language, the mechanic analysis software is developed which is applied in the well Jin 105-12X of Huabei oilfield.

\section{Acknowledgements}

University-enterprise cooperation projects from Huabei Oilfield Company of Petrochina (HBYT-CYY-2014-JS-192): Research on String Stress Analysis of Layered Iinjection-Production.

\section{References}

[1] Aijun Sun, Yingna Xu, Honglie Li, et al. Calculation of water injection string [J]. Drilling and Production Technology, 2003, 26(3): 55-57. (In Chinese )

[2] Jingyuan Li, Zifeng Li, Tianjiang Li, et al. Mechanical analysis software of downhole operation pipe strings and its application [J]. Petroleum Drilling \& Production Technology, 2008, 30(5): 118-121. (In Chinese ) 
[3] Zifeng Li, Hu Sun, Jinzhu Su, et al. Theory and applications of mechanical analysis of tubing string in fracturing operation [J]. Journal of Basic Science and Engineering, 2012, 20(5): 846-862. (In Chinese )

[4] Yanxin Liu, Hanxiang Wang, Jun Fang, et al. Mechanical analysis of injection string and its application [J]. Journal of Mechanical Strength, 2012, 6(1): 79-82. (In Chinese )

[5] Qi Liu, Jianxun Jiang, Qing Shi, et al. Calculation Analysis on force deformation of packer string with free movement [J]. Fault-Block Oil \& Gas Field, 2007, 14(1): 61-63. (In Chinese )

[6] Qindao Li, Guangping Xie, Juan Zhang. Mechanics and deformation analysis of immoveable packer string [J]. Drilling and Production Technology, 2002, 25(2): 53-57. (In Chinese )

[7] Jianxun Jiang, Qi Liu, Fuping Cheng, et al. Calculation of string deformation for separate zone water injection technique [J]. Journal of Southwest Petroleum University, 2007, 29(5): 145-148. (In Chinese )

[8] Yihua Dou, Fuxiang Zhang. Mechanical Analysis of well testing down-hole string in deep well with HTHP and its application [J]. Drilling and Production Technology, 2007, 30(5): 17-20. (In Chinese )

[9] Yanxin Liu, Hanxiang Wang, Naihe Hou, et al. Mechanical Analysis of oil test strings for deep wells and its application [J]. Drilling and Production Technology, 2012, 35(4): 71-73. (In Chinese )

[10] Jixian Leng. Underground column buckling behavior theory and experiment [D]. Nanchong, Sichuan: Southwest Petroleum University, 2003. (In Chinese )

Author introduction: Gang Mingchao, born in 1986, male, Hebei Cangzhou, in 2008, graduated in major mechanical engineering and automation from Tianjin Polytechnic University, now being engineer of Petroleum Engineering Research Institute of Huabei Oilfield Company. Email: cyy_gangmc@petrochina.com.cn. 